\title{
Aesthetics and Complexity in Digital Layout Systems
}

\section{Associate Professor Paul Cleveland}

Griffith University, Australia

p.cleveland@griffith.edu.au

\section{Abstract}

The layout of pages which carry information is the bread and butter of the graphic design profession. The rationale of what elements are chosen and where they are placed is based on tacit knowledge gained over a number of years of experience. The aesthetics associated with the overall finished design are often based on a style. Important elements of style are complexity and aesthetic value. In this paper I explore how various mathematical aesthetic functions can use complexity as an arousal enhancing or limiting mechanism in the application of layout designs.

\section{Keywords}

Aesthetics, Design, Layout, Complexity, Style

\section{Introduction}

Graphic designers employ a variety of methodologies in the process of discovering the best solution to a given problem from a client brief. Usually the solution, or set of solutions, can be seen to be working at a number of levels. Schmitt and Simonson's (1997) use a set of style dimensions consisting of complexity, representation, movement and potency to describe the domain of aesthetics as it is used in creating a visual grammar for company branding. Complexity here is used to denote the bipolar dimension of minimalism versus ornamentation. However, we will discover that high values of complexity relate to more than ornament. Representation is represented by the dimensions of realism versus abstraction, movement by the opposites of dynamic and static, and potency by strong and weak. The defining of style on these bipolar dimensions is not just about identifying qualities of these values, but also about their salience and significance. The order and organization of the elements, such as the colour, line and shape, are the basics of design, and differing styles have different grammars which equate to the rules around which they are governed.

The creation of a visual grammar is not just concerned with the aesthetic attributes of the layout, but rather with the visual relationships between all the elements that make up that layout. This includes images, their composition, the colours used, the quality of the reproduced image, use of type, spatial relationships, and many similar elements, as well as those that have been excluded. Aesthetic attributes in this sense is not concerned with beauty or good 
taste as a value, but difference in application. I make no judgements on the appreciation of one style over another, only that both are acceptable depending on the application and viewing audience.

The Schmitt and Simonson (1997) Style matrix is only one method which can be used to identify the perceptual dimensions of style. More complex systems have been described by Chen and Owen (1997), and Chan (2000). In all these systems the Style matrix attempts to construct style descriptions through the use of human observation to construct a measurable dimension.

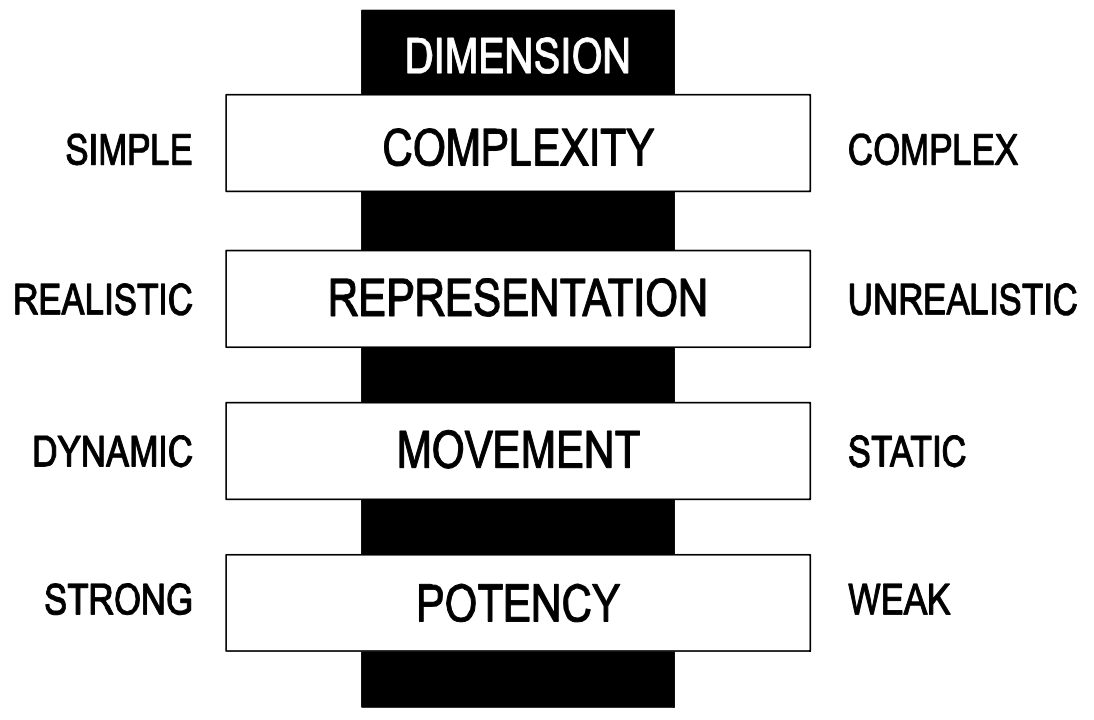

Figure 1. Dimensions of Style (After Schmitt \& Simonson 1997, p.113)

The simplicity of the Schmitt \& Simonson model allows a rudimentary examination of visual grammar into stylistic attributes. The model can be used by transferring a measured dimension into an algorithm for creating exemplars. Not all dimensions can be examined in an objective manner. For example, "representation" is a subjective dimension that can be measured by use of a semantic differential. When making an assessment of stylistic interpretation based on a period of time, mean counts of exemplars within a period would also give an acceptable result of stylistic trend. Movement and potency are linked to complexity in the layout placement of the elements that make up a design. This includes considerations relating to shape, orientation, number, and the class of elements. Take for example the images in Figure 2. The one on the left has lower values of movement and potency than the image on the right. This is due to the centre position of the text within the canvas, lack of colour, and the predominance of horizontal and vertical vectors indicated by the red arrows. The image on the right is predominated by angled vectors which not only increase the movement and potency, but also the complexity by adding opposing and conflicting passages for eye movement. We will take this idea as our starting point to 
explore complexity and how it can influence dimensions such as movement and potency.

\section{The Computational Notion of Complexity in layout systems}

Past research into automated layout systems tend to fall into two approaches. The first applies constraints to the layout in order to control the placement of elements (Lok et al 2004, Vander Zanden and Myers 1990, Borning and Duisberg 1986). The typical solution uses the concept of a design grid which has been adapted from traditional graphic design methodologies for print layout. The second approach uses a set of templates as exemplars of good design. These are often produced by design layout experts (Myers et al 1993). Most of these layout systems provide solutions whose aesthetic ideal is based on balance, equilibrium and symmetry. There is an assumption that these attributes represent "good" design. This is a classical notion based on the aesthetic valuation of the Classical Greeks and Romans. While there is undoubtedly some appeal in the order created by balance, equilibrium and symmetry, overuse or exposure encourages habituation and a decline in what Berlyne (1974) describes as arousal potential. The idea that aesthetic value is a combination of opposite factors which heighten or lower arousal has been known for some time. Berlyne (1974) gives an historical account of their existence. The two opposing states can be equated to harmony and tension; harmony being the "pleasing" arrangement of elements to generate a pleasing feeling, while tension equates to an arrangement which produces nervous or emotional strain.

Arousal restraining mechanisms are associated with familiarity, recognized groupings or patterns, and known expectations these generally equate to the harmony state. Designers and artists often use tension in layouts and pictures to create and promote greater interest, and this is accomplished through modifications to symmetry and balance. These arousal generating systems are recognized by their complexity using novelty, conflict and instability. The designs in Figure 2 illustrate this application, with the image on the right applying these attributes through the position and angles of the various elements.

Research into complexity associated with aspects of layout design originally tried to arrive at a numerical value which could show that the lower value of complexity equated to better design solutions because design is primarily concerned with creating order (Bonsiepe, 1968). Bonsiepe's (1968) methodology used a grid system to calculate distances from the page margins, and using Shannon's (1948) mathematical theory of communication to reinforce subjective observations of complexity. Shannon's entropy became Bonsiepe's measure of disorder. 
Tullis (1988) used Bonsiepe's mathematical formula to validate the effect of complexity on information retrieval. Tullis's work has subsequently been expanded by Comber and Maltby (1995), Ngo, Samsudin and Abdullah (2000), and $\mathrm{Ngo}$ and Byrne (2001). This avenue of research is concerned with providing some aesthetic measure based on evaluations of the properties of elements
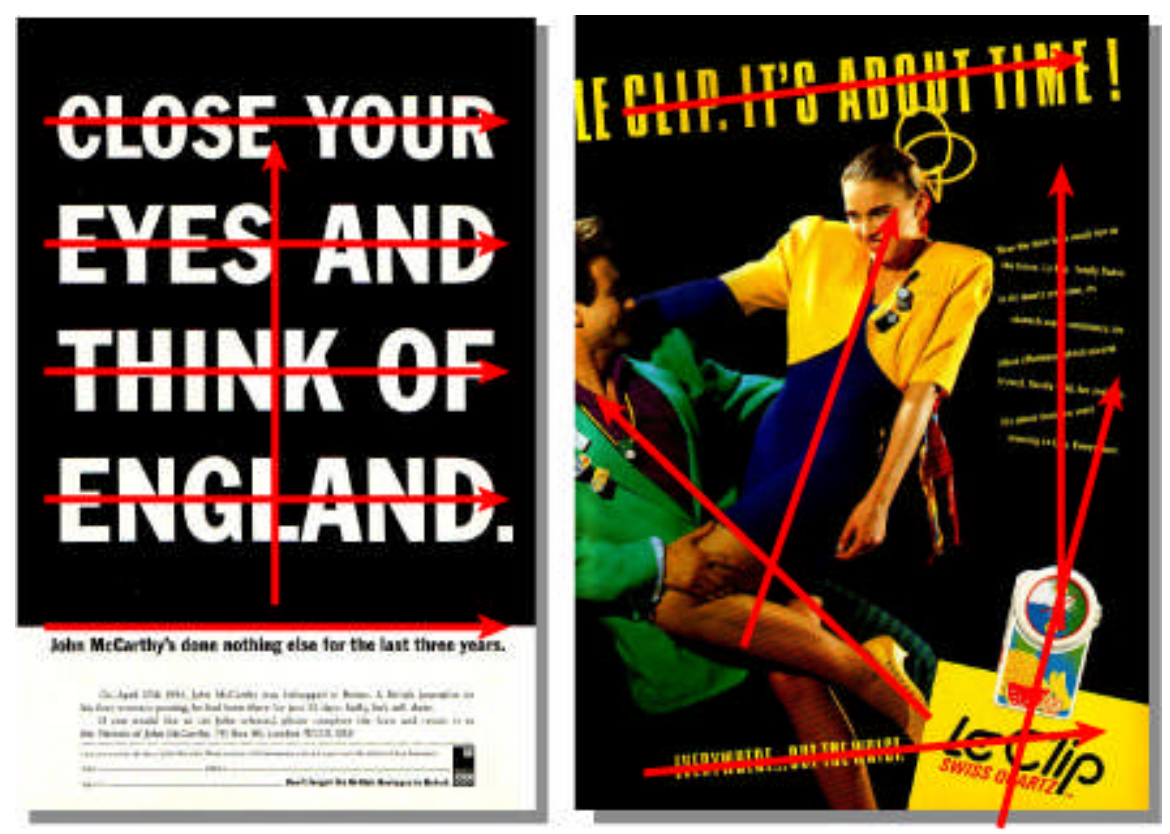

Figure 2. Example of the application of movement and potency which equates to complexity.

placed on a defined background. Ngo and Byrne (2001) identify eight different measures for the calculation of "aesthetic" values within a layout. These being balance, equilibrium, symmetry, unity, proportion, simplicity, density, economy, and order and complexity which is an aggregation of all the previous measures. The underlying assumption is that there are "good" and "bad" exemplars of these values. "Good" designs are characterized by high values. While there is logic to the choice of variables, graphic designers brought up in the western tradition are aware that there is a tendency for these types of solutions to appear conservative, dull and monotonous. The repeated exposure to such presentations eventually decreases the arousal potential. Such methodologies do not facilitate experimentation and development of novel layout solutions which are the hallmarks of the evolution of design styles over time. The aesthetic judgments represented by Ngo and Byrne (2001) represent only one aesthetic value and do not take into account visual aesthetics based on other stylistic attributions which create differing visual grammars.

Designers see complexity in terms of relationships between elements within a defined space. These relationships have various degrees of aesthetic quality depending on the audience viewing the piece of work. The aesthetic quality rests 
in "the eye of the beholder". If I was to design an expert system which would cater for all these possibilities I would need to formulate a methodology for describing differences in aesthetics and devise a visual grammar which could reproduce that aesthetic. It is this visual grammar that can be interpreted as a design style.

Designers have used various measures to evaluate the aesthetics of designs. Lavie and Tractinsky (2004) identify empirical studies of aesthetics as falling into two categories. The first looks at the individual elements of a design, and makes an attempt to rationalize particular qualities which can then be taken as universal truths concerning aesthetics. Typically this includes the research of Berlyne (1974) who investigated responses to shapes and patterns, and Martindale (1986) who investigated observed responses to art. The second category is concerned with an individual's perception of an evaluated object and is therefore subjective in nature. Taking the first of these as a starting point we can devise a style based ontology which describes rules associated with that particular style aesthetic. The measurement of complexity and it's association with aesthetics would be an important consideration.

\subsection{Aesthetics and proportion}

The use of proportion as an aesthetic measure has a long history. The Pythagorean interpretation of beauty was said to exist in proportions. There have been numerous empirical investigations over the past 130 years examining the aesthetic qualities of shapes containing the Golden Section proportion (Green, 1995). The outcomes range from evidence of general trends toward some form of preference, to no effect at all. Green concludes that there are "real psychological effects associated with the golden section, but they are relatively sensitive to careless methodological practices" (Green, 1995 p.937).

The use of the Golden Section in itself would be of little use as an aesthetic measure of communication design, but it can be used as a method for reducing arousal. This may seem strange as we have noted that there is a preference for this type of ratio. This preference we shall see is based on a lack of arousal. Berlyne (1970) found that preference toward the Golden Section was a western phenomenon and other cultures such as Japanese had a preference for the square. Berlyne (1971) makes reference to German psychologist Frank (1959, 1964) who theorized that it was the Auffalligkeit ("strikingness" or "penetrance") which makes a design attractive, and to maximize this effect requires one to maximize the information it contains. Frank's formulation for "strikingness" related to the frequency of the information $i$, denoted $\mathrm{p}_{\mathrm{i}}$, and the information content of $\mathrm{i}$, given by $-\log \mathrm{p}_{\mathrm{i}}$. The measure for "strikingness" is the product of these two measures, that is, $-p_{i} \log p_{i}$. This formula is the same as that presented by Shannon (1948) in his seminal publication on communication theory. It can be 
shown that quantity of $-p_{i} \log p_{i}$ peaks at 0.368 , which is close to the minor ratio of the Golden Section (Figure 3).

\begin{tabular}{|lll|}
\hline pi & $-\log \mathrm{pi}$ & - pi log pi \\
0.02 & 2.322 & 0.464 \\
0.38 & 1.396 & 0.530 \\
0.60 & 0.737 & 0.442 \\
\hline
\end{tabular}

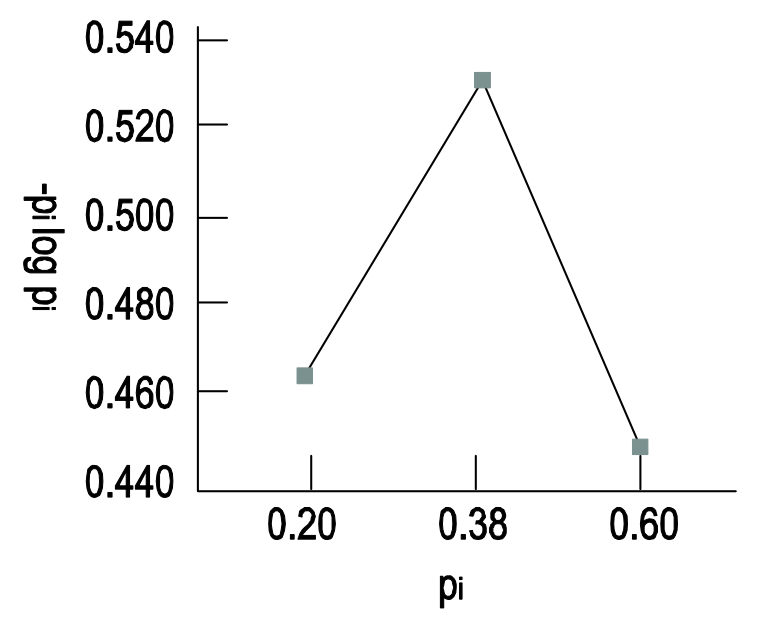

Figure 3. The "strikingness" measure reaches a maximum when $\mathrm{p}_{\mathrm{i}}=\mathbf{0 . 3 8}$.

Frank produced some examples by a range of artists which in which paintings contained a proportion of a colour which came close to a Golden Section ratio. Taking Frank's proposition that artists use this ratio to increase the "strikingness" of their artwork, a similar experiment was conducted.

\subsection{Hypothesis}

Aesthetic preference involves complex factors which optimise the degree of arousal potential. The use of ratios may be one of these, but balance, complexity and order are also important factors. The use of the Golden Section will show neither high nor low values in harmony or complexity but would be regarded as generally agreeable.

\subsection{Materials}

Mondrian's geometric compositions are based on grid structures which closely resemble grids used by graphic designers when laying out page designs. The analysis of these images using a semantic differential as well as digital image analysis can easily determine if any Golden Section ratios were used either intentionally or unintentionally, and determine preferences based on the structure and composition of the image. Piet Mondrian 1872-1944 by Yve-Alain Bois and Joop Joosten, published by Bulfinch Press (1994), was chosen as it contains 
good reproductions of Mondrian's work in a chronological sequence, and the images are deemed to be exemplary representations of the artists work. Eight sequential images were selected because they represented a good survey of his geometric work and closely relate to grid layouts used by graphic designers. The works follow in sequence from plate 88 to plate 95 . Eight works were deemed sufficient to gauge an overall impression of application.

In all there were four procedures to the experiment. The first required the design of a web site which solicited respondents' opinions on the Mondrian images through the use of a semantic differential. The second determined the optical centre of balance in the image. The third determined the colour area within each image, and the fourth matched saliency points of each image with Golden Section proportions.

\subsection{Respondents}

In the fourth procedure a web site wad designed to catch respondents' answers to a set of bipolar scales. There were 30 respondents, 12 female and 18 male aged between 16 and 50. The respondents were all readers of Graphic Design forums and responded to a posted invitation to participate.

\subsection{Procedure}

The first procedure required each image (Figure 3 ) to be scanned in colour at 200 dpi. An algorithm to find the optical centre was applied to each image. The optical centre is important as it tends to focus the eye's attention.

The optical centre of an image is the average location of its weight, or equivalently, the location at which the object's weight can be assumed to be concentrated. As darker objects appear heavier than light ones, we define the weight of a colour to be the darkness value of the colour, scaled to a value between 0 (lightest) and 1 (darkest) inclusive. If one considers an image as simply an $h$ by $w$ array of pixels (indexed from the top left corner of the image) such that each pixel $p_{i j,} 0 \leq i \leq h, 0 \leq j \leq w$, has weight $w t\left(p_{i j}\right)$, then we define the optical centre of an image to be the pair $\left(C_{X}, C_{Y}\right)$ where

$$
C_{X}=\frac{\sum_{i=0}^{h-1}\left(\sum_{j=0}^{w-1} i \times w t\left(p_{i j}\right)\right)}{\sum_{i=0}^{h-1}\left(\sum_{j=0}^{w-1} w t\left(p_{i j}\right)\right)} \quad \text { and } \quad C_{Y}=\frac{\sum_{j=0}^{w-1}\left(\sum_{i=0}^{h-1} j \times w t\left(p_{i j}\right)\right)}{\sum_{j=0}^{w-1}\left(\sum_{i=0}^{h-1} w t\left(p_{i j}\right)\right)} .
$$

This formula is based on the well know formula (Halliday et al, 2001) for determining the centre of mass of a set of particles (Cleveland \& Jenkins, 2006). 

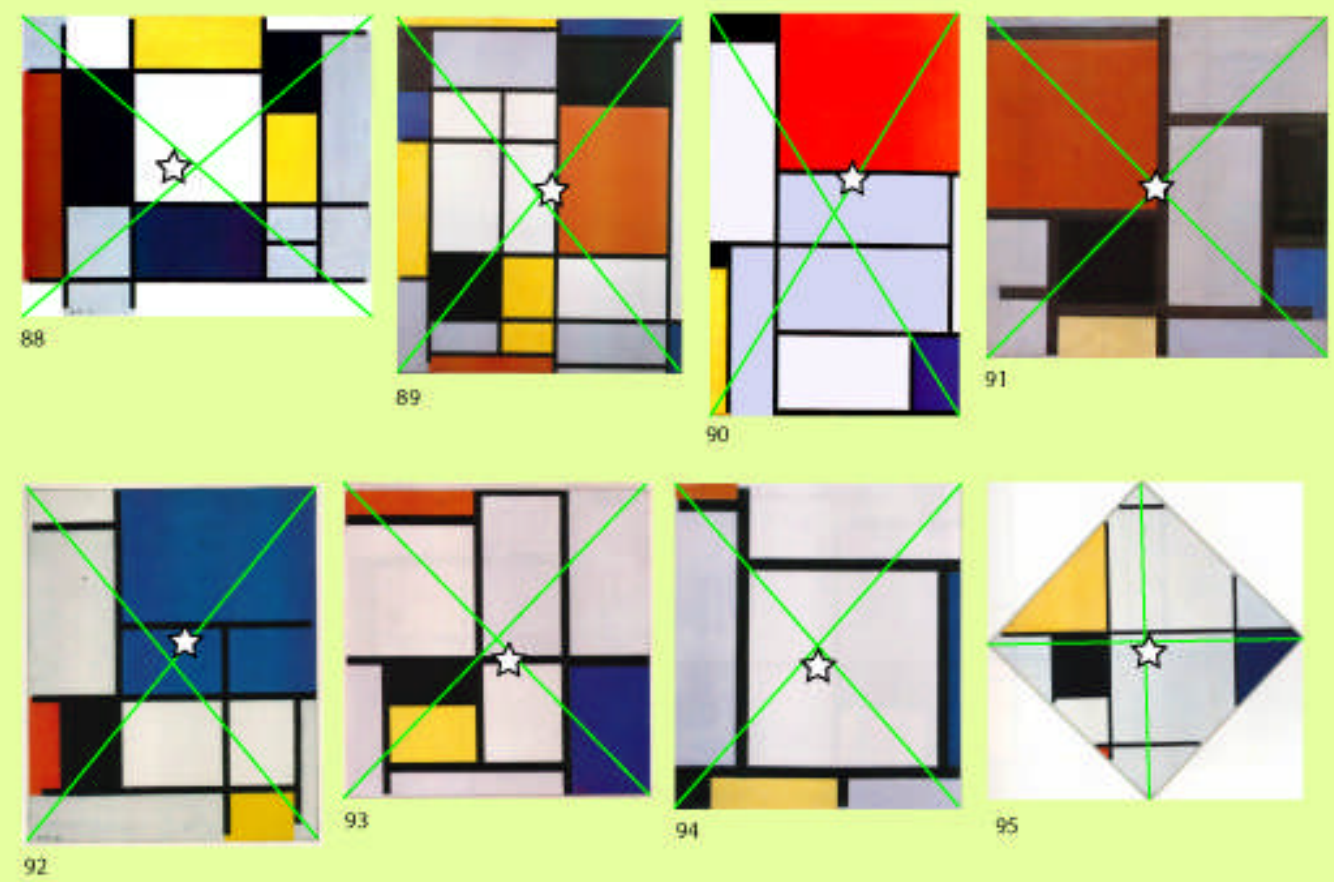

Figure 4. The Mondian pictures used in the experiment. The star indicates the optical centre and the green lines the geographic centre.

The second procedure required the calculation of the percentage of each colour used in each composition. Each image was placed in University of Texas' software ImageTool. Images were converted to gray scale and a threshold filter applied to isolate each colour from the image. When a colour was isolated a pixel count was applied. The data was then converted into a percentage of overall pixels.

In the third procedure a perceptual saliency tool processed each image. The development of a computer based perceptual saliency tool has made significant progress in recent years. An understanding of the process of determining visual attention within visual imagery is needed to appreciate its value in determining saliency. It is generally recognized that there are two processes at work when the act of attention focusing is engaged, although there is still debate over whether one process is sufficient or both need to be activated to achieve visual consciousness (Itti and Koch, 2000). The first is the slower top down mechanism "with variable selection criteria, which directs the 'spotlight of attention' under cognitive, volitional control" (Itti and Koch, 2000). The other known as the bottom up process is a "primitive mechanism that biases the observer towards selecting stimuli based on their saliency (most likely encoded in terms of centre-surround mechanisms" (Itti and Koch, 2000).

Although there are a number of computational models developed to detect saliency, the Itti and Koch (2001) model shows remarkable application to 
detection of saliency in design. Based on the Koch and Ullman (Koch and Ullman, 1985) model for bottom up visual attention it uses a saliency map to identify saliency locations and was stimulated by the behaviour and neural architecture of early primate visual systems. This process breaks up the complexities involved in viewing imagery through the use of algorithms which analyse information. The input image is analysed for features such as colour, intensity, orientation, and motion, in all 42 maps are generated. The saliency maps are then combined into one map, see Figure 5. This method of extracting saliency is based on spatial competition where a particular feature (Winner Takes All, WTA) wins one or more spatial scales. Progressive saliency can also be computed by suppressing the original location and detecting the next most salient position. This process is known as the "Inhibition of Return" (Itti, Koch and Niebur, 1998).

The fourth procedure required respondents to indicate their opinions of the pictures on eight scales. These scales were;

Harmonious - Discordant

Simple - Complex

Orderly - Disorderly

Weak - Powerful

Ugly - Beautiful

Like - Dislike

Balanced - Unbalanced

Tense - Relaxed

A seven point rating scale allowed respondents to indicate the degree to which an image contained an attribute. The scales were selected to measure four domains; those of balance, complexity, order and personal taste. The address of the online test was posted on five Graphic Design forums asking those interested in design and aesthetics to fill out the survey. The responses were then automatically emailed back to the researcher who compiled the results and used a two way analysis of variance (picture (8) X scales (8)) to establish if there were any statistically significant results. In all there were 30 responses. 

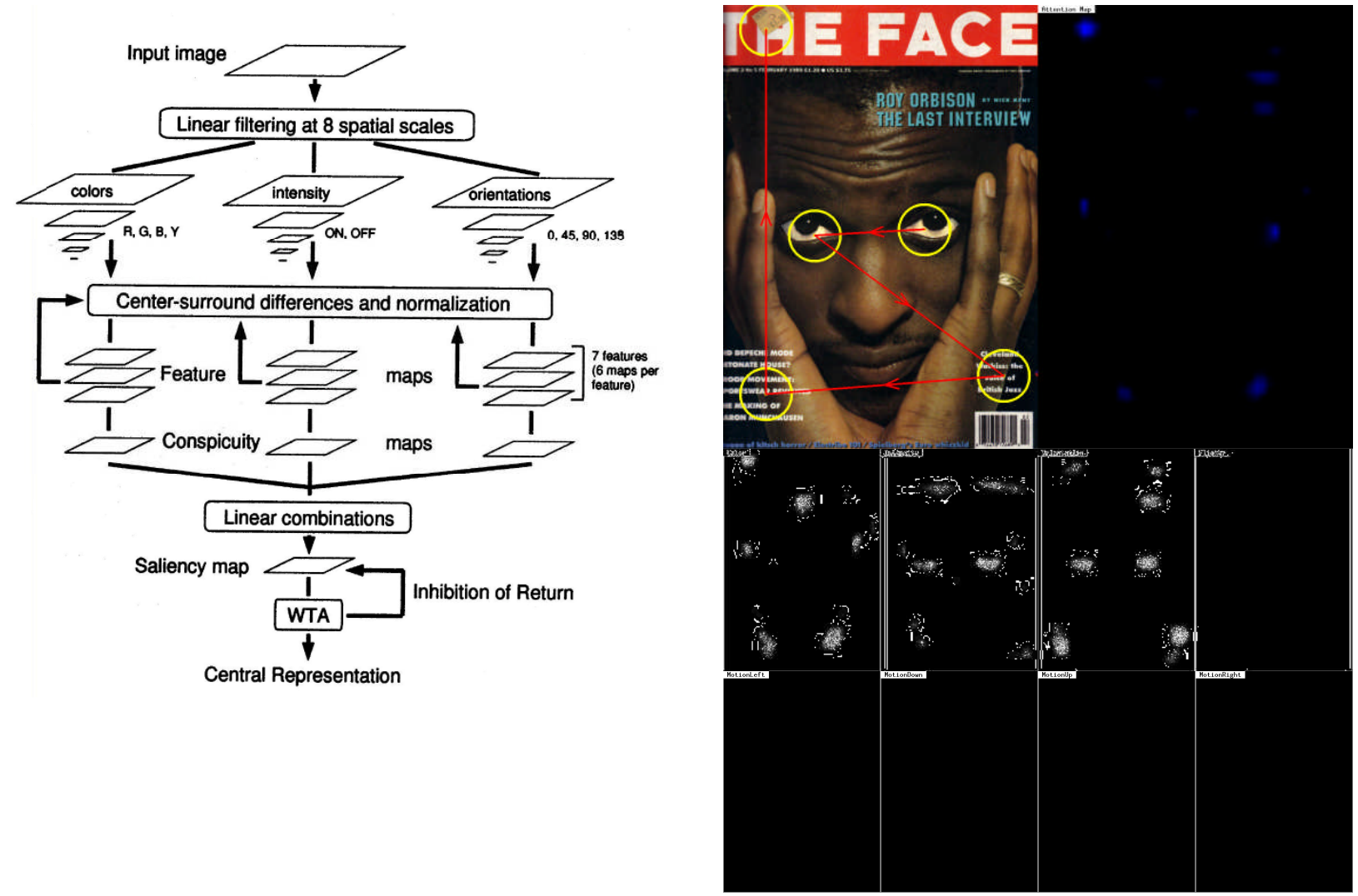

Figure 5. Left: A diagram of the bottom up saliency process (Itti, Gold, Koch, 2001). Right: Result of an image after processing.

\subsection{Results}

The results for procedure one can be seen as the red dot in Figure 4. The geographical centre can be seen as the intersection of the green lines.

The results for procedure two can be seen in Figure 6 which illustrates the pixel counts for each processed image. The percentages which approximate the two ratios of the Golden Section are 38 and 62. Close values (within five percentage marks) for single colours close to 38 occur 4 times, in picture 91, 92, 93 and 95. There are no recorded values that come close to 62 . This would suggest that the occurrence is more by chance than deliberate intent.

Picture 88

$\begin{array}{llll}\text { White } & 28.78 \% & \text { White } & 26.21 \% \\ \text { Yellow } & 10.46 \% & \text { Yellow } & 9.41 \% \\ \text { Gray } & 22.36 \% & \text { Gray } & 16.51 \% \\ \text { Red } & 8.28 \% & \text { Red } & 17.78 \% \\ \text { Blue } & 9.25 \% & \text { Blue } & 5.08 \% \\ \text { Black } & 21.05 \% & \text { Black } & 25.02 \%\end{array}$

Picture 90

$\begin{array}{ll}\text { L/ Gray } & 22.64 \% \\ \text { Yellow } & 2.41 \% \\ \text { D/Gray } & 32.80 \% \\ \text { Red } & 28.04 \% \\ \text { Blue } & 3.83 \% \\ \text { Black } & 10.29 \%\end{array}$

Picture 91

$\begin{array}{ll}\text { L/ Gray } & 40.83 \% \\ \text { Yellow } & 3.40 \% \\ \text { Red } & 24.80 \% \\ \text { Blue } & 3.05 \% \\ \text { D/Gray } & 15.56 \% \\ \text { Black } & 12.18 \%\end{array}$




\begin{tabular}{llllllll}
\multicolumn{2}{l}{ Picture 92} & \multicolumn{2}{l}{ Picture 93} & \multicolumn{2}{l}{ Picture 94} & \multicolumn{2}{l}{ Picture 95} \\
L/ Gray & $13.61 \%$ & L/ Gray & $26.36 \%$ & L/ Gray & $56.84 \%$ & L/ Gray & $28.83 \%$ \\
D/Gray & $28.10 \%$ & Yellow & $5.17 \%$ & Yellow & $4.22 \%$ & D/Gray & $38.44 \%$ \\
Yellow & $3.12 \%$ & D/Gray & $37.37 \%$ & D/Gray & $21.07 \%$ & Yellow & $11.54 \%$ \\
Red & $3.04 \%$ & Red & $3.80 \%$ & Red & $1.13 \%$ & Red & $0.73 \%$ \\
Blue & $37.24 \%$ & Blue & $11.08 \%$ & Blue & $2.89 \%$ & Blue & $37.24 \%$ \\
Black & $14.90 \%$ & Black & $16.20 \%$ & Black & $13.85 \%$ & Black & $14.90 \%$
\end{tabular}

Figure 6. Colour pixel counts for each image.

Results for procedure three are illustrated in Figure 7. Analysis with the perceptual saliency tool indicated saliency points on each image. Points were limited to six as this number was thought to be sufficient to gauge compliance within, or outside the Golden section. The yellow circles represent saliency points and the sequence can be gauged by the direction of the arrows on the connecting lines. The pink bands indicate the Golden Section ratios both horizontal and vertical (Figure 7). The line width is set at the saliency circle size. Hits were labelled either direct if they were close to the centre of the Golden Section band, or peripheral if they straddled the edge of the band. Figure 8 shows the results in tabulated form. Only picture 94 showed no direct hit, only a peripheral one. Again all pictures except 94 obtained a combined hit rate of $50 \%$ or better. Intentionally or unintentionally the designs of the pictures show sympathy for the ratios in question. The results of this research do not discount that there maybe other aesthetic ratios used in the compositions.

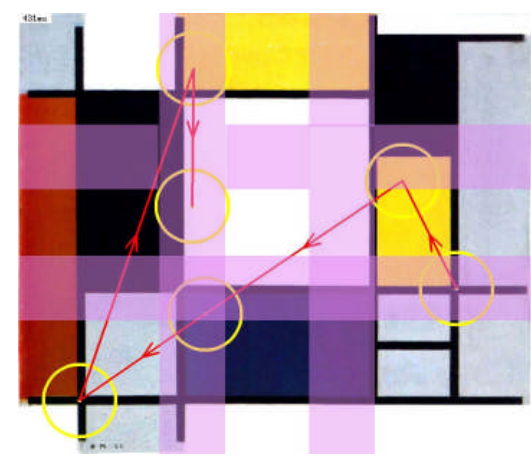

88

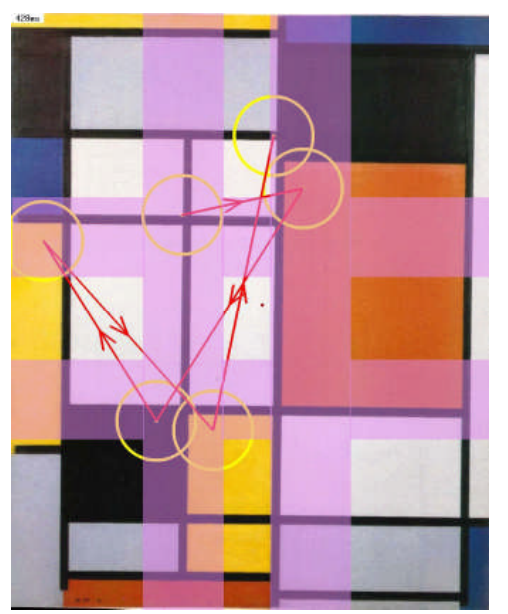

89

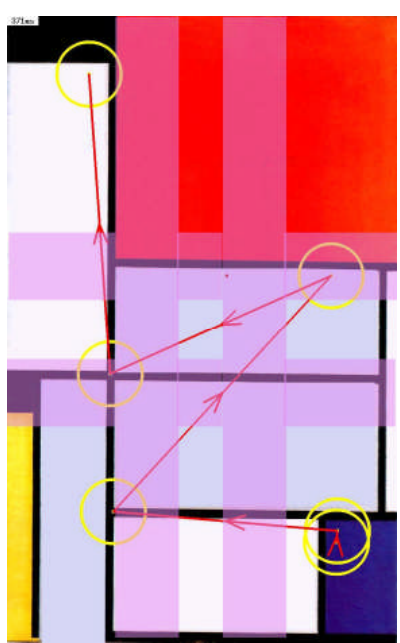

90 


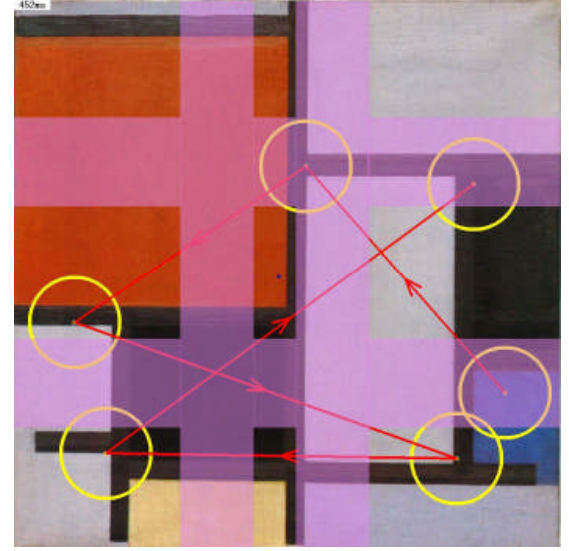

91

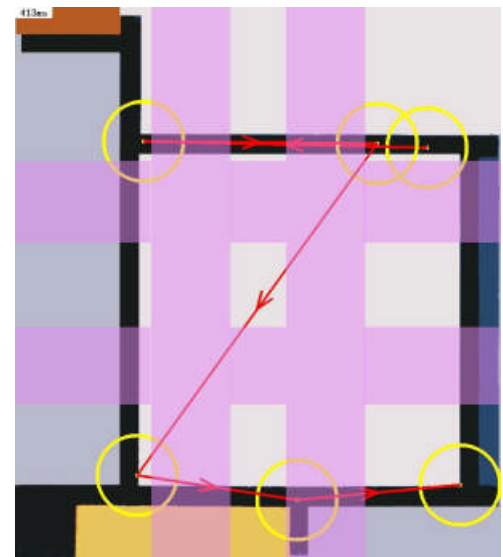

94
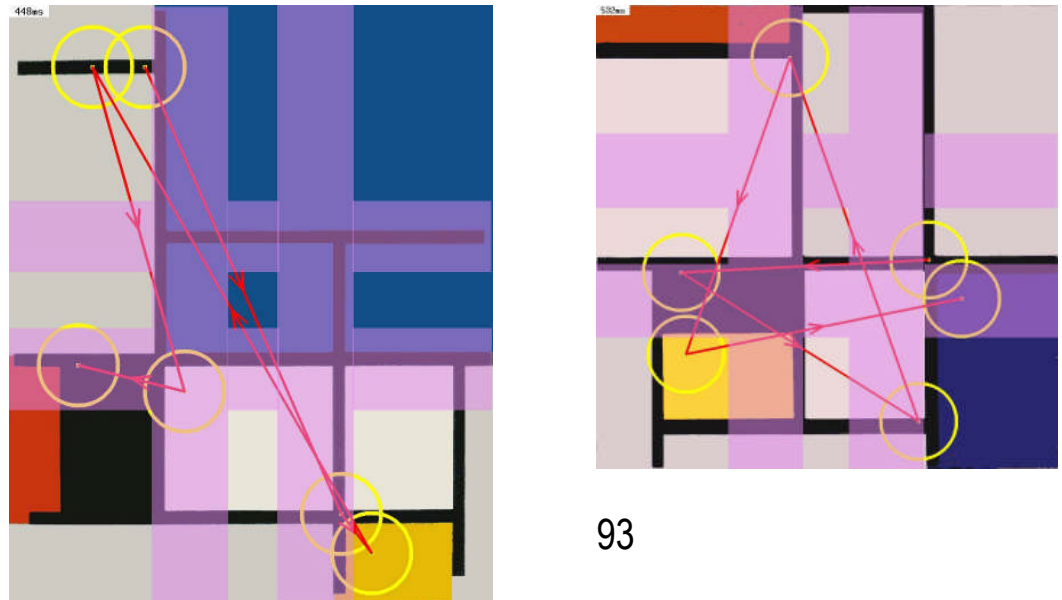

93

92

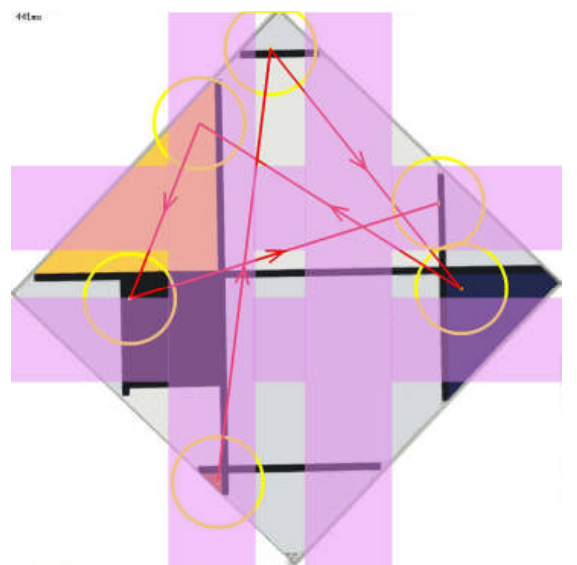

95

Figure 7. Results from saliency analysis.

$\begin{array}{lll}\text { Image } & \text { Direct Hits (First) } & \text { Peripheral Hits } \\ 88 & 4 \text { (Yes) } & 1 \\ 89 & 3 \text { (Yes) } & 3 \\ 90 & 1 & 2 \\ 91 & 2 \text { (Yes) } & 1 \\ 92 & 2 & 1 \\ 93 & 1 & 3 \\ 94 & 0 & 1 \\ 95 & 3 \text { (Yes) } & 0\end{array}$

Figure 8. Golden Section hits. First refers to the first saliency point in the run being a direct hit. 
Results for procedure four indicated that the mean values among the responses for each image were greater in some scales than would be expected by chance at $(P=<0.001)$; these scales were harmonious/discordant, simple/complex, orderly disorderly, balanced/unbalanced, and tense/relaxed.

\subsection{Discussion}

The domain of personal taste found no statistical difference in preference between the images. However picture 91 recorded the highest score for like with a mean value of 3.733 (Figure 9). The next in preference was 89 and 93 which both scored 4.033. The results for procedure one identified 91 as the only image where the optical and geographic centre corresponded. 91 was also found to be statistically more harmonious than other images with a mean of 2.993. This image also recorded the highest value for orderly and rated equal with 93 for most relaxed. A comparison with results from procedure two shows that it has a light grey pixel count of $40.83 \%$ which is close to the Golden Section. Procedure three also indicates a first direct hit on the Golden Section. The scales of harmony, orderly and tense may be influenced by the Golden Section ratio which is evident in the construction of the image.

Comparisons were made with 92,93 and 95 to see if there was any corresponding evidence, as these had received Golden Section percentages in procedure two. Image 92 and 95 were seen as discordant while 93 received the second highest value for harmonious. Therefore there was no consistency in evidence of the use of the Golden Section producing a consistent value for harmony. While 93 was considered orderly 92 and 95 were considered more disorderly with a statistical difference in the means of 1.433. Mean values for tense showed similar results with 93 regarded as relaxed but 92 and 95 recording higher values for tense. The conclusion was that percentage of colour with values close to the Golden section showed no consistency in the scales used to measure the domains of balance, complexity, order and personal taste.

Comparisons were then made using procedure three, which tracked the saliency to the Golden Section proportions of the images. The images with the greatest number of direct hits were compared using the data gathered from procedure four; these being 88,89 and 95 . All three recorded a mean value with no statistical difference between 3.967 and 4.200. For simple, all recorded means with no statistical difference between 3.567 and 3.900 , this would rate them as neither simple nor complex. The orderly scale again showed no statistical difference between the images in question with means ranging from 3.233 to 3.733. On the weak scale again there was consistency with means ranging from 3.967 to 4.333 with no statistical difference between results. In the domain of personal taste the results for ugly and like showed no statistical difference. The 

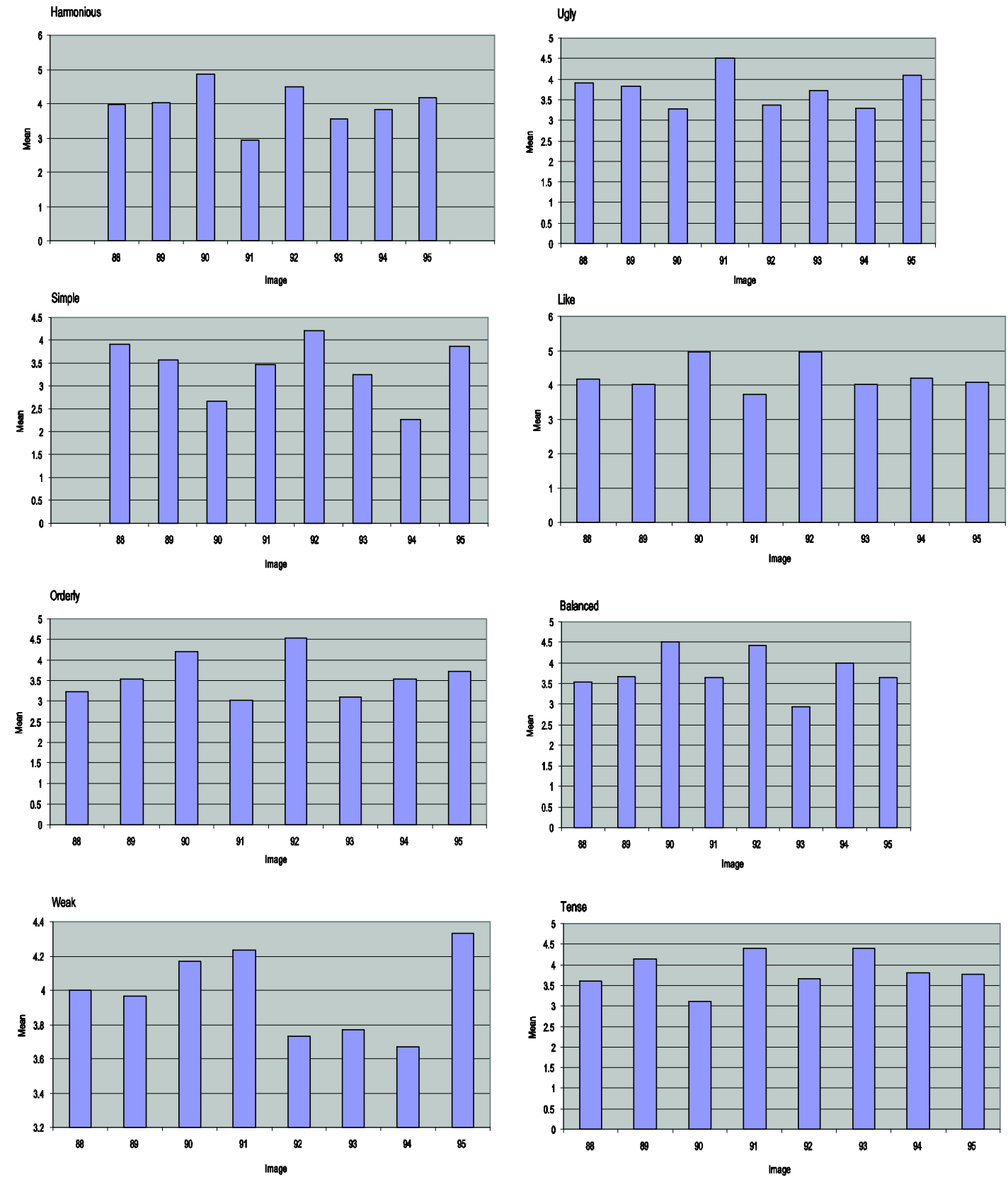

Figure 9. Mean values for images and scales.

results for balance and tense had similar results with no statistical difference recorded.

The results from this exercise show that there is a greater match between Golden Section ratios dividing a page than percentage of colour. The characteristics associated from the assignment of such ratios as determined from this experiment are the following. Images tend to be neither harmonious or discordant. They could be described as agreeable. They are neither simple nor 
complex but tend to be orderly. These images are regarded as balanced but neither tense nor relaxed. It all amounts to a kind of neutral position. Compare this with the results for an image which had no percentage of colour, or ratio close to the Golden Section. Image 90 fits this category. From the results of procedure four this image would be described as simple in structure, discordinant and disorderly. With these traits it rates high on the powerful scale and is disliked but not deemed to be ugly. It is seen as unbalanced and conveys a sense of tenseness. These are the descriptors associated with producing an increase in arousal.

The conclusion drawn from this study is that the use of a ratio like the Golden Section in a design has a tendency to fall into Berlyne's (1971) description of an arousal restraining mechanism. I believe that Berlyne's translation of the word Auffalligkeit as incorrect. Other translations are remarkableness, distinctiveness, conspicuous and salience. So the reason why Golden Section images have these attributes is because they are perceived as more agreeable or satisfying rather than striking, which tends to indicate larger measures of arousal.

The use of ratios such as the Golden Section, as well as the use of symmetry can be used as moderating devices when constructing style based layouts. The term style based layout is used because the formation of a solution to a digital layout problem needs to pass through a design layout ontology which can set solution up as styles for repeated use. Before we come to that stage we need a methodology for converting proportions into a layout system.

\section{Birkhoff's notion of complexity}

Berlyne's (1974) geometric studies acknowledge the work of Birkhoff (1933), who was also interested in the aesthetic qualities of geometric shapes. Rather than looking at proportion as a measure of aesthetic value, Birkhoff chose to concentrate on visual sensations that were created by observing key features of a form. Birkhoff identified these features as being order and complexity. The "strickingness" of Frank (1959) can be translated into "effort of attention" which can increase in proportion, this Birkhoff called "complexity" (C) (Birkhoff, 1950).

... a realization that the object is characterized by a certain harmony, symmetry, or order (0), more or less concealed, which seems to be necessary for the aesthetic effort (Birkhoff, 1950, p.321).

The aesthetic measure is then determined by "the density of order relations in the aesthetic object" (Birkhoff, 1950). The formula $\mathrm{M}=\mathrm{O} / \mathrm{C}$ would then produce an aesthetic measure which could compare objects within particular classes.

Birkhoff's formula has been used as a basis for a number of studies associated with the application of the formula to particular design issues (Staudek 1999, 
Ngo, Samsudin and Abdullah, 2000, Ngo, Teo, and Byrne, 2002). Birkhoff's original study of polygonal shapes calculated order from vertical symmetry (V), equilibrium $(E)$, rotational symmetry $(R)$, close relation to the horizontal-vertical network (HV), all which were positive values and unsatisfactory form $(\mathrm{F})$ which was a negative value and consisted of a number of attributes which were considered to be "ambiguity of forms" or general lack of symmetry or ambiguity (Birkhoff, 1933). The formula for $O$ therefore is $O=V+E+R+H V-F$.

Birkhoff (1933) discussed the various ratios and their aesthetic qualities based on the proportions derived from the square, these being:

1.414 which when divided produces a rectangle of the same proportion.

\subsection{8 the Golden Section}

\subsection{2 based on a double equilateral triangle}

2 based on the double square

All these proportions have their own aesthetic qualities which Birkhoff measured using the above formula. The sum of vectors that described a shape became the measure of complexity. This is fine for the study of polygonal shapes but needed some modification for use in layout design.

The use of vectors as a measure of complexity can be transferred to graphic design layouts and measured in a similar way. You will recall the use of vectors in figure 2 to describe complexity. To illustrate this approach a set of simple elements has been used to construct exemplars. The set consists of one illustration, one text paragraph and four individual lines of text (Figure 10). The relationship between the elements is as follows, with the lower number being the most salient. The elements in 4 all have similar rankings.

1. Masthead - "Field \& Farm"

2. Illustration

3. Contents - Contents paragraph

4. 4.1 By-line - "The magazine for Countrymen"

Date - "January 1958"

Price - "Three Shillings" 
There are a number of constraints imposed on the text elements and these relate to perceived importance as judged by the designer. This example represents a design for a magazine cover and traditional design logic places the text relationships in order of saliency. Figure 10 represents two of the solutions based on a low calculation for complexity. The one on the left has high values of symmetry and equilibrium while the image on the right has low values in symmetry but high values in equilibrium. The examples here have been generated using "Processing" software to illustrate a proof of concept.
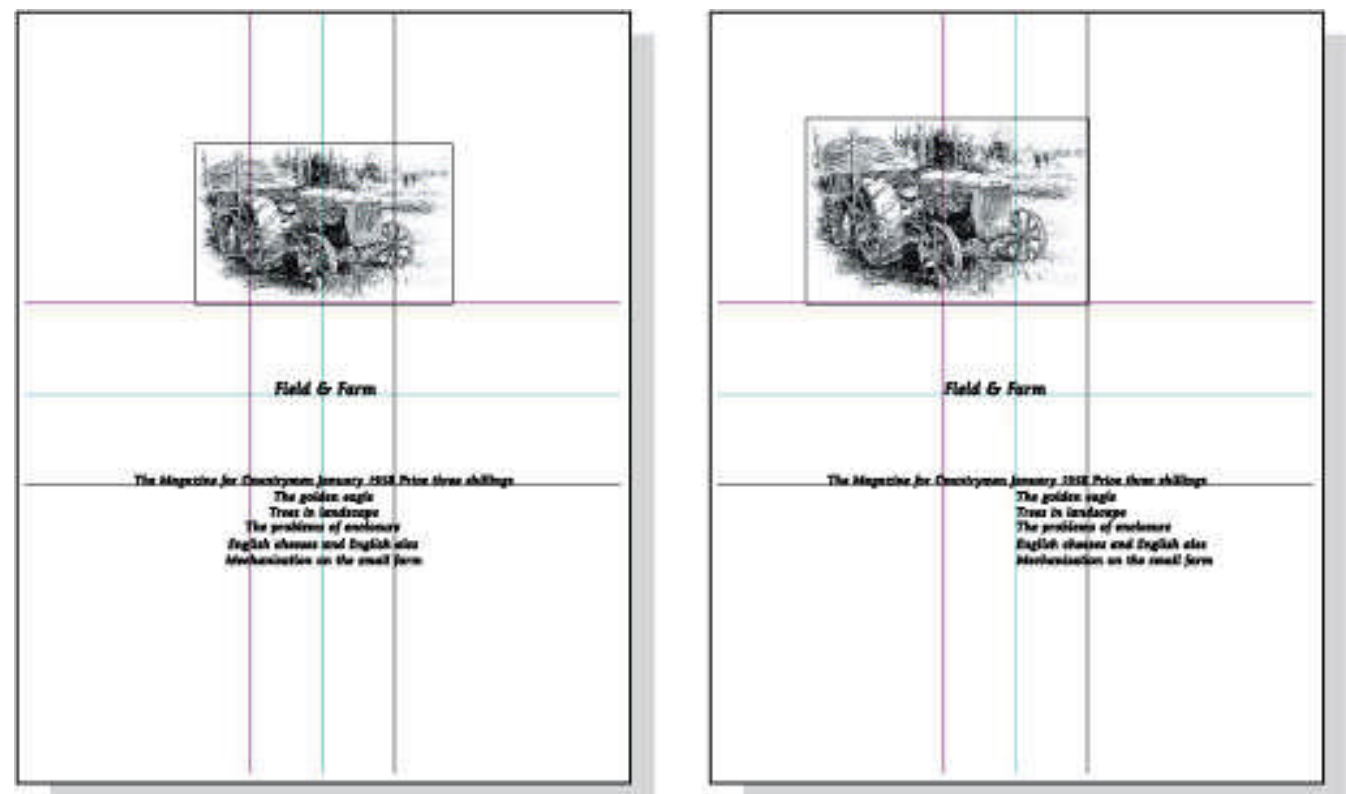

Figure 10. Left: $C=3+1+3+0=7$, Right: $C=3+3+4+0=10$

The formula is based on the least number of horizontal and vertical extended lines which define the alignment of the elements. The example in Figure 10 uses the ratio of the Golden Section to produce the grid division. Typically text is aligned on the first baseline of a block of text, and left, right or centrelines govern vertical alignment. This assigns a value for the complexity of placement but does not address issues associated with the number of differing element variables. For example, the introduction of a different type weight, font style or point size will increase the complexity of the design. To accommodate these issues the formula also takes into account the number of differing elements. Complexity is the sum of $(\mathrm{H})$ horizontal grid lines used, plus sum of $(\mathrm{V})$ vertical grid lines used, plus the number of different elements, plus the number of different treatments.

$\mathrm{C}=\sum_{i=1}^{n} H+\sum_{j=1}^{n} V+\sum_{k=1}^{n} E+\sum_{k=1}^{n} T$

Therefore for the left layout in Figure 10 the calculation would be $C=3+1+3+0=7$. This represents a measure of complexity and is not related to a measure of good or bad design. Rearranging and resizing the elements can produce a more 
complex design which has greater communication value due to clustering of the elements, as in Figure 11.
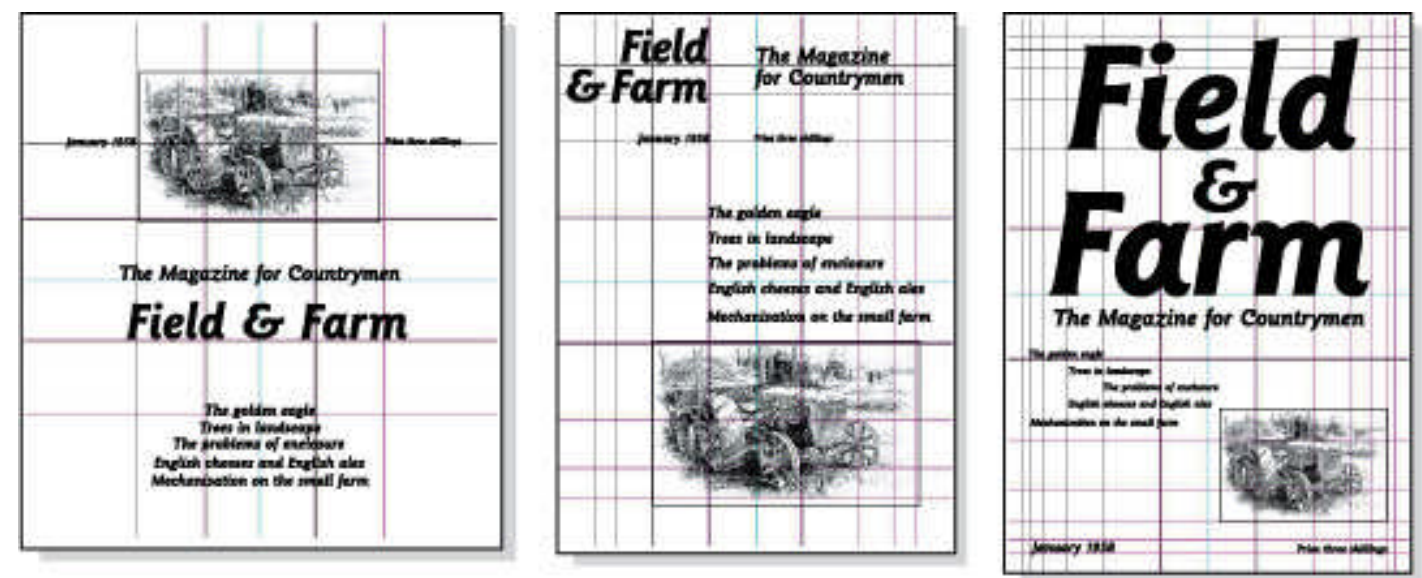

Figure 11. Complexity values using different Golden Section grids, from left to right grids of 3,5 and 7 with corresponding complexity values of 17,17 and 19.

It can be seen that complexity values can produce a range of solutions. The more horizontal and vertical grid lines used the more complex the design will be. However there is no guarantee that all permutations will produce satisfactory results. While this methodology produces numerous satisfactory results they are characterized by low values of arousal potential because of the visual similarity of many of the solutions due to the limited font and image library.

The logic associated with constructing the layout is based on a semantic differential which accepts responses from the user (Figure 12). The logic set out in Figure 12 illustrates two sets of relationships which exist between the elements which make up the design. The text elements have a child-parent relationship and saliency is indicated through a hierarchical structure. The two solutions illustrated show the results of the choices made in the semantic differential. The solution on the left is after a harmonious, simple, weak, colourless, balanced geometric result. The logic for this result looks at each of the bipolar differentials and formulates a result related to the intensity of the selection. A high value for harmony was chosen, which equates to the use of the Golden Section and central divisions of the page space into which to set the elements. Harmony also necessitates that all objects must sit within the edge limits of the page with no element edge touching the page edge, or cropping off the page. The opposite of this can be seen in the solution on the right where the word "FARM" leaves the edge of the page. This image also shows discordant alignments of elements at various angles. Simple and complex are defined in a number of ways; firstly by the number of grid options, and secondly by the number of elements and treatments. The weak/powerful dimension looks at the relationship between the elements to determine if they are exaggerated or not. Compare the two examples 
in Figure 13 which are basically the same apart from the differences in the weak/powerful dimension.


Figure 12. Composition logic.

The bipolar dimensions for colour determine the amount of colour and the palette used in the design. High values of colour look at contrasting colours according to hue value rather than complimentary colour. Balance is determined by the two values of symmetry and equilibrium. High values of balance equate to the use of both symmetry and equilibrium while the opposite does not use symmetry and purposefully places elements out of equilibrium. The dimensions of geometric and organic look at the placement of elements along horizontal and vertical alignments for high values of geometric or more random placement for organic. Organic graphics have non geometric features and freeform edges.

\section{Conclusion}

In this paper I have tried to explain how a methodology based on aesthetics and complexity can be used as a basis for determining generative layouts. The use of ratios such as the Golden Section which are generally regarded as having an arousal enhancing effect actually operate in the opposite way. What I have put forward are only general principals. The development of styles in design has a number of more complex dimensions associated with the elements which are the 
building blocks of the design. The research described in this paper will aid in the formulation of a more comprehensive structure to the construction of visual grammar and associated styles.



Harmonious 01020304050607 Discordant Simple 01020304050607 Complex Weak 01020304050607 Powerful Colourful 01020304050697 Colourless Balanced 01020304050607 Unbalanced Geometric 01020304050607 Organic

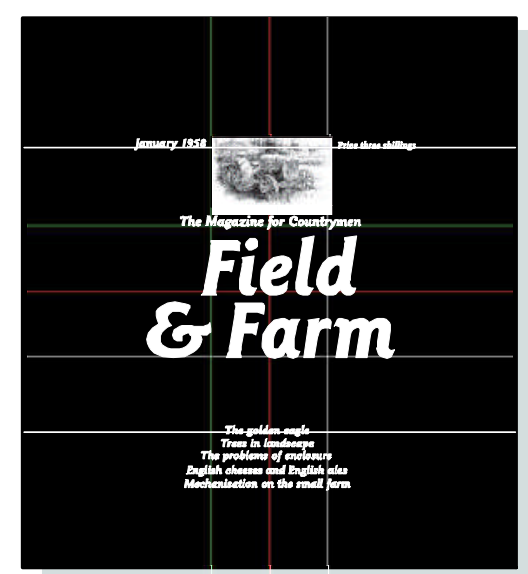

Harmonious 1020304050607 Discordant Simple 01020304050607 Complex Weak 01020304050607 Powerful Colourful 01020304050607 Colourless Balanced 01020304050607 Unbalanced Geometric 01020304050607 Organic

Figure 13. Comparison of the weak/powerful dimension.

\section{References}

Berlyne, D. E. (1970). "The golden section and hedonic judgments of rectangles: A cross-cultural study", Sciences de l'art/Scientific Aesthetics, 7, 1-6.

Berlyne, D.E. (1971). Aesthetics and Psychobiology, Appleton-Century-Crofts, Meredith Corporation, New York.

Berlyne D.E. (Ed.) (1974). Studies in the new experimental aesthetics, Washington, Hemisphere Publishing.

Birkhoff G. D. (1933). Aesthetic Measure, Cambridge, Harvard University Press.

Birkhoff G. D. (1950). Collected Mathematical Papers, Volume III, New York, American Mathematical Society.

Bois, Y. and Joosten, J. (1994). Piet Mondrian, 1872-1944. Bulfinch Press, Boston.

Bonsiepe, G.A. (1968). "A Method of Quantifying Order in Typographic Design", Journal of Typographic Research, Vol. 2.

Borning, A. and Duisberg R. (1986). "Constraint Based Tools for building User Interfaces”, ACM Trans. On Graphics, 5(4). 
Chan, C. (2000). "Can style be measured?", Design Studies, 21, (3).

Chen, K., \& Owen C. (1997). "Form language and style description", Design Studies, 18, (3).

Cleveland, P.; Jenkins, P. (2006). Unpublished paper, "Complexity in Generative Solutions to Style Based Designs", http://kali.qcas.gu.edu.au/qcacleve/sites/research.html, online November 2006.

Comber, T and Maltby, J. R. (1995). "Evaluating usability of screen designs with layout complexity", OZCHI '95: Proceedings of the CHISIG Annual Conference, Melbourne, Australia.

Ngo, D. C. L.; Byrne, J. G. (2001). "Application of an Aesthetic Evaluation Model to Data Entry Screens", Computers in Human Behavior, v17 n2 p14985.

Frank, H. (1959). Grundlagenprobleme der Informationsästhetik und erste Anwendung auf die mime pure [Fundamental problems of information aesthetics and the first application to the mime pure.], Quickborn: Schnelle.

Frank, H. (1964). Kybernetische Analysen subjektiver Sachverhalte [Cybernetic analyses of subjective conditions]. Quickborn: Schnelle.

Green C. D. (1995). "All That Glitters: A Review of Psychological Research on the Aesthetics of the Golden Section", Perception, 24, 937-968.

Halliday, D., Resnick, R., \& Walker, J. (2001). Fundamentals of Physics, 6th Edition. Wiley, New York.

Itti. L, Kock, C. and Niebur. E, (1998). "A Model of Saliency-Based Attention for Rapid Scene Analysis", IEEE Transactions on Pattern Analysis and Machine Inteligence, Vol. 20, No.11, November.

Itti, L, \& Kock, C. (2000). "A saliency-based search mechanism for overt and covert shifts of visual attention", Vision Research 40.

Koch, C., \& Ullman, S. (1985). "Shifts in selective visual attention: Towards the underlying neural circuitry", Human Neurobiology, 4.

L. Itti, C. Koch, (2001). "Computational Modeling of Visual Attention", Nature Reviews Neuroscience, Vol. 2, No. 3. 
Lavie T. \& Tractinsky N. (2004). "Assessing dimensions of perceived visual aesthetics of web sites", International Journal of Human Computer Studies, 60, pp. 269-298, Elsevier.

Lok, S., Feiner, S. and Ngai, G. (2004). Evaluation of Visual Balance for Automated Layout, IUI'04, [online], citeseer.ist.psu.edu/660520.html.

Martindale, C. (1986). "The Evolution of Italian Painting: A Quantitative Investigation of Trends in Style and Content from the Late Gothic to the Rococo Period", Leonardo. 19, (3), 217-222.

Myers, B.A., McDaniel, R.G. and Kosbie, D.S. (1993). "Marquise: Creating compete user interfaces by demonstration", Proc. INTERCHI '93, Human Factors in Computer Systems.

Ngo, D. C. L., Samsudin, A. and Abdullah, R. (2000). "Aesthetic Measures for Assessing Graphic Screens", Journal of Information Science and Engineering 16, 97-116.

Ngo, D. C. L., Teo, L. S., and Byrne, J. G. (2002). "Evaluating Interface Aesthetics", Knowledge and Information Systems 4: 46-79.

Schmitt B. \& Simonson A. (1997). Marketing Aesthetics, New York, The Free Press.

Shannon, C.E. (1948). "A Mathematical Theory of Communication", The Bell System Technical Journal, Vol.27, [online], http://cm.belllabs.com/cm/ms/what/shannonday/shannon1948.pdf.

Staudek T. (1999). "On Birkhoff's Aesthetic Measure of Vases", FI MU Report Series, Faculty of Informatics, Masaryk University, [online], http://www.fi.muni.cz/reports/files/older/FIMU-RS-99-06.pdf.

Tullis, T.S. (1988). "Screen Design", Handbook of Human-computer Interaction, Helander, M. (Ed.), Elsevier Science Publishers B.V., Amsterdam.

Vander Zanden, B. and Myers, B.A. (1990). "Automatic, look-and-feel indipendent dialogue creation of graphical user interfaces", Proc. ACM CHI'90 Conference on Human Factors in Computer Systems. 